\title{
TRIVIAL POINTS ON TOWERS OF CURVES
}

\author{
XAVIER XARLES
}

\section{INTRODUCTION}

When studding the solutions of families of diophantine equations, there are some (usually called) trivial solutions. Moreover, one would like to know if for all members of the family with, may be, a finite number of excepcions, the only solutions are the trivial ones. In this paper we would like to study what should be a trivial solution for the case that the family forms a tower. The idea is that the trivial solutions are the solutions that are always there, so they should be points that exists at all the levels of the tower. And our main goal will be to find conditions in order to show when there is a finite number of such trivial points, and also when there are uniform bounds for the level of the tower where all the points are trivial.

The main example to have in mind is the case of the modular towers: consider the curves $X_{1}\left(p^{n}\right)$ for some fixed prime $p$, and $n \geq 0$. Then the trivial points should correspond to the cuspidal points. And, moreover, there is a constant $N(p, d)$ depending on $p$ and $d \geq 1$ such that, for any number field $K$ with $[K: \mathbb{Q}] \leq d$, and for any $n \geq N(p, d)$, the only $K$-rational points of $X_{1}\left(p^{n}\right)$ are the trivial ones (see for example [26]).

Another example was considered by the author in [31]; the result is similar to the case of modular curves. This paper is, in some sense, a sequel of that paper where we investigate under which circumstances one can get this type of results.

There are other recent papers treating similar problems. For example in the paper [10] it is studied cases similar to the modular towers, related to the inverse Galois problema (see also [18] for other generalizations), and in [14] it is studied also such (vary general) cases but for families indexed by prime numbers.

The paper is organized as follows. In section 2 we introduce the towers and their trivial points, and give some elementary results. In section 3 and 4 we study the special cases of towers with genus 0 and 1 , giving some partial results. In section 5 we give a criterium for proving the finiteness of trivial points of a tower. In section 6 we recall the well-known relation between the unbounded gonality and the existence of uniform bounds (see Theorem ??). The rest of the sections are dedicated to the distinct methods to bound the gonality of a tower: geometric methods, reduction modulo primes and counting points, and to methods related to graphs.

This paper contains results concerning towers of curves that the author collected during some years. The content was explained during the "Cuartas Jornadas de Teoría de Números" in Bilbao, in July 2011. I would like to thank the organizers for the invitation to give a talk, which motivated me to write this paper. I thank also Enrique González Jiménez, Joan Carles Lario, Francesc Bars, Pete Clark, and Brian Conrad, for some conversations related to the

The author was partially supported by the grant MTM2009-10359. 
subject. I specially thank Bjorn Poonen for answering some doubts concerning the gonality and for the help proving some results in section 3 .

\section{Notations, Generalities And EXAmples}

Given a field $K$, we will denote by $\bar{K}$ a fixed separable closure of $K$ and by $G_{K}$ the absolute Galois group of $K$, equal to $\operatorname{Aut}_{K}(\bar{K})$, the automorphisms of the field $\bar{K}$ fixing the elements of $K$. Given any scheme $V$ over $K$, we will denote by $\bar{V}$ the base change of $V$ to $\bar{K}$.

Definition. Let $K$ be a field. By a tower of curves over $K$ we mean a couple of sequences $\mathrm{C}:=\left(\left\{C_{n}\right\}_{n \geq 0},\left\{\varphi_{n}\right\}_{n \geq 1}\right)$, where, for any $n \geq 0$, a positive integer, $C_{n}$ are smooth projective algebraic curves defined over $K$ and geometrically connected, and, for any strictly positive integer $n>0$, the $\varphi_{n}: C_{n} \rightarrow C_{n-1}$ are non constant morphisms as algebraic curves, of degree $>1$ for all $n \geq 0$.

If $m>n$, we will denote by $\varphi_{m, n}$ the morphism from $C_{m}$ to $C_{n}$ obtained composing the morphisms from $\varphi_{m}$ to $\varphi_{n+1}$. We will denote also $\varphi_{n, n}$ the identity map on $C_{n}$.

Definition. Given two towers of curves $\mathbf{C}=\left(\left\{C_{n}\right\}_{n \geq 0},\left\{\varphi_{n}\right\}_{n \geq 1}\right)$ and $\mathbf{C}^{\prime}=\left(\left\{C_{n}^{\prime}\right\}_{n \geq 0},\left\{\varphi_{n}^{\prime}\right\}_{n \geq 1}\right)$, a morphism $\Psi$ from $\mathbf{C}$ to $\mathbf{C}^{\prime}$ is a collection of morphisms $\psi_{n}: C_{n} \rightarrow C_{k_{n}}^{\prime}$ such that $k_{n} \leq k_{m}$ and $\varphi_{k_{n}, k_{m}}^{\prime} \psi_{n}=\psi_{m} \varphi_{n, m}$ if $n \leq m$. A special case is when $\mathbf{C}$ is a subtower of $\mathbf{C}^{\prime}$, i.e $C_{n}=C_{k_{n}}^{\prime}$ and $\psi_{n}$ is the identity, for some progression $k_{0}<k_{1}<k_{2}<\ldots$

Given a smooth projective algebraic curve $C$ over a field $K$, we will denote by $g(C)$ the genus of $C$, and by $\gamma(C)$ the gonality of $C$ over the field $K$ (see section 5 for details).

Definition. Given a tower of curves $\mathbf{C}$, we define the genus $g(\mathbf{C})$ to be $\lim \sup g\left(C_{n}\right)$, and the gonality $\gamma(\mathbf{C})$ as $\lim \sup \gamma\left(C_{n}\right)$.

Given a tower of curves, we have by Hurwitz's theorem that $g\left(C_{n}\right) \geq g\left(C_{n-1}\right)$ for any $n$, so $0 \leq \lim \sup g\left(C_{n}\right)=\lim g\left(C_{n}\right) \leq+\infty$. The same fact for the gonality is not so easy; one can find a proof of this result for example in Proposition A1 in [29] (see also section 6).

Lemma 2.1. For any tower $\mathbf{C}$, the genus can only be $g(\mathbf{C})=0,1$ or $\infty$.

Proof. This is again due to Hurwtiz: if there exists a curve $C_{n}$ in the tower with genus $g\left(C_{n}\right)>1$, then $g\left(C_{m+1}\right)>g\left(C_{m}\right)$ for all $m \geq n$, so $g(\mathbf{C})=\infty$.

Example. One can construct genus 0 towers easily by fixing rational functions $f_{n}(x) \in K(x)$ of degree $>1$. Over algebraically closed fields, all genus 0 towers are of this type (see section $3)$.

Also, to construct genus 1 towers, one can fix elliptic curves $E_{n}$ and isogenies $\varphi_{n}: E_{n} \rightarrow$ $E_{n-1}$. An easy example is given when $E_{n}=E$ and $\varphi_{n}$ is multiplication by some fixed integer number for all $n \geq 0$ (see section 4 ).

Definition. Given a tower of curves $\mathbf{C}=\left(\left\{C_{n}\right\}_{n \geq 0},\left\{\varphi_{n}\right\}_{n \geq 1}\right)$ defined over $K$, the $K$-trivial points of $\mathbf{C}$ in the level $n \geq 0$ are

$$
\mathbf{C}(K)_{n}:=\left\{P \in C_{n}(K) \mid \forall m \geq n \exists P_{m} \in C_{m}(K) \text { such that } \varphi_{m, n}\left(P_{m}\right)=P\right\} .
$$

If $d \geq 1$ is an integer, the $K$-trivial points of $\mathbf{C}$ in the level $n \geq 0$ and degree $d$ are

$$
\mathbf{C}^{(d)}(K)_{n}:=\bigcup_{\substack{L \subset \bar{K},[L: K] \leq d \\ 2}} \mathbf{C}(L)_{n} .
$$


Finally, the trivial points of $\mathbf{C}$ in level $n$ are

$$
\mathbf{C}^{(\infty)}(K)_{n}:=\bigcup_{d \geq 1} \mathbf{C}^{(d)}(K)_{n} .
$$

So the trivial points in level $n$ are the points that are $L$-trivial in level $n$ for some finite extension $L / K$.

In the case of level 0 we will frequently omit it from the notation.

Observe that over an algebraically closed field the trivial points of a tower of curves in the level $n$ is equal to all the rational points of the curve $C_{n}$. So these definitions are only interesting in fields such as number fields, or, more generally, fields finitely generated over the prime fields.

Observe also that the $K$-rational trivial points in level $n$ are equal to the imatge in $C_{n}(K)$ of the natural map

$$
\lim _{\overleftarrow{n}} C_{n}(K) \rightarrow C_{n}(K)
$$

where the projective limit is taken with respect to the maps $\varphi_{n}$.

The main problem we are going to analyze is when there are a finite number of $K$-trivial points, and when a finite number of trivial points. The next lemma is easy and we leave the proof to the reader.

Lemma 2.2. Let $\mathbf{C}=\left(\left\{C_{n}\right\}_{n \geq 0},\left\{\varphi_{n}\right\}_{n \geq 1}\right)$ be a tower of curves over some field $K$.

(1) For any $d=0,1, \ldots, \infty$, the natural map $\varphi_{n, m}: \mathbf{C}^{(d)}(K)_{n} \rightarrow \mathbf{C}^{(d)}(K)_{m}$ is surjective.

(2) For any $d=0,1, \ldots, \infty, \mathbf{C}^{(d)}(K)_{n} \subseteq \varphi_{n, m}^{-1}\left(\mathbf{C}^{(d)}(K)_{m}\right)$.

(3) Two isomorphic towers have natural bijections between their trivial points.

(4) If $\mathbf{C}^{\prime}$ is a subtower of $\mathbf{C}$, i.e. $C_{n}^{\prime}=C_{k_{n}}$ and $\psi_{n}$ is the identity, for some progression $k_{0}<k_{1}<k_{2}<\ldots$, then $\mathbf{C}^{(d)}(K)_{n}=\mathbf{C}^{(d)}(K)_{k_{n}}$ for all $n \geq 0$ and all $d=0,1, \ldots, \infty$.

We end this section by given some specific examples of towers of curves.

Example. Consider a prime number $p$. We will denote by the $p$ Fermat tower the tower with curves $C_{n}$ given by planar homogeneous equations $X_{0}^{p^{n}}+X_{1}^{p^{n}}=X_{2}^{p^{n}}$, and maps $\varphi_{n}\left(X_{0}, X_{1}, X_{2}\right)=\left[X_{0}^{p}: X_{1}^{p}: X_{2}^{p}\right]$. One can show that the only trivial points of the tower are the "trivial solutions" $\left[a_{0}: a_{1}: a_{2}\right]$ with $a_{0} a_{1} a_{2}=0$.

Example. Consider a prime number $p$. We will denote by the $p$ modular tower the tower with curves $C_{n}:=X_{1}\left(p^{n}\right)$ and natural maps $\varphi_{n}$. One can show (see section 5 ) that the only trivial points of the tower are the cuspidal points.

Example. Consider the homogeneous polynomial $f_{0}:=X_{0}^{2}+X_{1}^{2}-X_{2}^{2}$, and $C_{n} \subset \mathbb{P}^{n+2}$ be the curves defined over $\mathbb{Q}$ as the zero set of the polynomials $f_{0}, f_{1}, \ldots, f_{n}$, where $f_{n}:=$ $f_{0}\left(X_{n}, X_{n+1}, X_{n+2}\right)$. For any field $K$, the $K$-rational points of $C_{n}$ are in bijection with the Fibonacci type sequences of squares of length $n+2$, that is sequences $\left\{a_{0}, a_{1}, a_{2}, \ldots, a_{n}\right\}$ such that $a_{n+2}=a_{n+1}+a_{n}$ and all the elements $a_{i}$ are squares in $K$. We will call this tower the Fibonacci tower.

Observe that we have four points $[ \pm 1,0, \pm 1,1] \in C_{1}(\mathbb{Q})$. One can show that $C_{1}$ is isomorphic to the elliptic curve $E$ with Cremona Reference $32 \mathrm{a} 2$, and that $E(\mathbb{Q})$ has only four points. So $C_{1}(\mathbb{Q})$ is form by that four points, thus $C_{2}(\mathbb{Q})=\emptyset$ and, hence, $\mathbf{C}(K)_{0}=\emptyset$. 
Using the results in section 6 one can show that the degree 2 points over $\mathbb{Q}$ of the curve $C_{2}$, which has genus 5 and gonality 4 (see section 7 ), inject inside the jacobian $\operatorname{Jac}\left(C_{2}\right)$. Using results as in [19, 20], one can show that the jacobian is isogenous to the product of 5 elliptic curves, with Cremona references 32a2,32a2,48a1,96a1 and 96b1. All of them have rank 0 and four rational points, $\operatorname{so} \operatorname{Jac}\left(C_{2}\right)$ is finite and computable. Using this result one gets that

$$
C_{2}(\mathbb{Q})^{(2)}=\{[\sqrt{-1}, \pm 1,0, \pm 1, \pm 1],[ \pm 1,0, \pm 1, \pm 1, \sqrt{2}]\}
$$

which can be use to show that $C_{3}(\mathbb{Q})^{(2)}=\emptyset$ and hence $\mathbf{C}(K)_{0}^{(2)}=\emptyset$. We do not know if $\mathbf{C}(K)_{0}^{(d)}=\emptyset$ for some $d \geq 3$ (but we do know these sets are finite using the results in section 6) or if $\mathbf{C}(K)_{0}^{\infty}=\emptyset$, or even finite.

Conjecture 2.3. The Fibonacci tower has no trivial points over any number field. Hence, the curves given by the system of equations

$$
X_{0}^{2}+X_{1}^{2}=X_{2}^{2}, X_{1}^{2}+X_{2}^{2}=X_{3}^{2}, \ldots, X_{n}^{2}+X_{n+1}^{2}=X_{n+2}^{2}
$$

inside $\mathbb{P}^{n+2}$ have no rational points for any number field $K$ and for $n$ large enough (in terms of the degree of $K / \mathbb{Q})$.

\section{Genus 0 Towers}

Consider a tower of curves $\mathbf{C}$ with genus 0 . It is well known that a genus 0 curve is either isomorphic to the projective line $\mathbb{P}^{1}$ (and if and only if it has a rational point in your field), or isomorphic to a conic curve (see for example Theorem A.4.3.1. in [21]). In this second case, there exists some degree 2 extension of the field where the curve gets isomorphic to $\mathbb{P}^{1}$ (and, even, there are infinitely many such degree 2 extensions if the field is a number field).

Definition. Given an enumerated set $\mathcal{F}:=\left\{f_{n}(x) \in K(x)\right\}_{n \geq 1}$ of rational functions with degree $>1$, consider the tower of curves $\mathbf{C}_{\mathcal{F}}$ defined as $C_{n}:=\mathbb{P}^{1}$ and $\varphi_{n}=f_{n}(x)$ for all $n \geq 0$. The special case that $f_{n}=f$ for all $n$ will be denoted by $\mathbf{C}_{f}$.

The next lemma was communicated to me by Bjorn Poonen.

Lemma 3.1. Let $C$ and $C^{\prime}$ be genus 0 curves over a number field $K$, and $f$ be a non-constant morphisms from $C$ to $C^{\prime}$. Suppose that $C^{\prime}(K)=\emptyset$. Then $C$ and $C^{\prime}$ are isomorphic (and the degree of $f$ is odd).

Proof. It is well known that the genus 0 curves $C$ without $K$-rational points correspond to conics without points, so to quaternion algebras over $K$, hence they give elements $x_{C}$ of order 2 in the Brauer group $\operatorname{Br}(K)$ of $K$. The existence of the map $f$ says us that $C$ has no rational points. We will see that $\operatorname{deg}(f) x_{C}=x_{C^{\prime}}$ in $\operatorname{Br}(K)$, hence $\operatorname{deg}(f)$ is odd and $x_{C}=x_{C^{\prime}}$, so $C$ is isomorphic to $C^{\prime}$. Observe that we have a natural map

$$
\mathbb{Z} \cong \operatorname{Pic}(\bar{C})^{G_{K}} \rightarrow H^{1}\left(G_{K}, \frac{\bar{K}(C)^{*}}{\bar{K}^{*}}\right) \rightarrow H^{2}\left(G_{K}, \bar{K}^{*}\right)=\operatorname{Br}(K)
$$

sending 1 to $x_{C}$, given by the natural connecting homomorphisms, which is functorial. On the other hand, the natural map $\operatorname{Pic}\left(\bar{C}^{\prime}\right)^{G_{K}} \rightarrow \operatorname{Pic}(\bar{C})^{G_{K}}$ is the multiplication by the degree of $f$. Hence the result is deduced from the commutativity of the natural diagram, which is easy.

As a consequence of this lemma and the results cited above, we get the following classification. 
Lemma 3.2. Let $K$ be a number field, and let $\mathbf{C}$ be a tower of curves. Then

(1) If $\mathbf{C}(K)_{0} \neq \emptyset$, then there exists a set $\mathcal{F}:=\left\{f_{n}(x) \in K(x)\right\}_{n \geq 1}$ of rational functions and an isomorphism $\mathbf{C} \cong \mathbf{C}_{\mathcal{F}}$.

(2) There always exists infinite degree 2 extensions $L / K$, an enumerated set $\mathcal{F}:=\left\{f_{n}(x) \in\right.$ $L(x)\}_{n \geq 1}$ and an isomorphism $\mathbf{C}_{L} \cong \mathbf{C}_{\mathcal{F}}$ defined over $L$.

Proof. If $\mathbf{C}(K)_{0} \neq \emptyset$, then there are points $P_{n} \in C_{n}(K)$ for all $n$, hence all $C_{n}$ are isomorphic to $\mathbb{P}^{1}$. But then the maps $\varphi_{n}$ give us endomorphisms of $\mathbb{P}^{1}$, so rational funcions $f_{n}$. The same is true if $C_{n}(K) \neq \emptyset$ for all $n \geq 0$.

Now, suppose there exists $n \geq 0$ such that $C_{n}(K)=\emptyset$. Using the previous lemma, we get that $C_{m}$ is isomorphic to $C_{n}$ for all $m \geq n$, and all isomorphic to a fixed conic $C$. Now we only need to recall that for any fixed conic $C$ over a number field there are an infinite number of quadratic extensions $L / K$ such that $C(L) \neq \emptyset$.

Now we are going to study the finiteness of $K$-trivial points. Hence we can and will assume that $\mathbf{C}(K)_{0} \neq \emptyset$. We will only get some partial results, using the theory of heights.

Remark. For the towers of the form $\mathbf{C}_{f}$, with $f(x) \in K(x)$, observe that $\mathbf{C}(K)_{n}=\mathbf{C}(K)_{0}$ for all $n$, and it contains the set of periodic points of $f$ : the points $P \in \mathbb{P}^{1}(K)$ such that $f^{N}(P)=P$ for some $N \geq 1$ (see [21], B.4, for example).

Theorem 3.3. Let $K$ be a number field and let $f(x) \in K(x)$ be a rational function of degree $d>1$. Then the set of $K$-rational trivial points of level 0 of the tower $\mathbf{C}_{f}$ is finite, and equal to the set of periodic points of $f$.

Proof. We will show that the set of $K$-rational points is equal to the set of periodic points, and finiteness will follow. Consider the canonical height function $h_{f}$ associated to $f$ (see for example [21], Theorem B.4.1.). Then $h_{f}(f(P))=d h_{f}(P)$ for any $P \in \mathbb{P}^{1}$, where $d>1$ is the degree of $f$. If $P \in \mathbf{C}_{f}(K)_{0}$, there exists $P_{n} \in \mathbb{P}^{1}$ such that $f^{n}\left(P_{n}\right)=P$, so $h_{f}(P)=$ $h_{f}\left(f_{n}\left(P_{n}\right)\right)=d^{n} h_{f}\left(P_{n}\right)$. Now, suppose that $h_{f}(P) \neq 0$. Then $h_{f}\left(P_{n}\right)=d^{-n} h_{f}(P) \leq h_{f}(P)$ are all distinct, so the set $\left\{P_{n} \mid n \geq 0\right\}$ is an infinite set of points of $\mathbb{P}^{1}$ with bounded canonical height $h_{f}$, hence with bounded absolute (logarithmic) height, which is not possible (see [21], theorem B.2.3). So $h_{f}(P)=0$ and hence $P$ is preperiodic by Proposition B.4.2.(a) in [21]. Now, if $P$ is preperiodic but not periodic, the set $\left\{P_{n} \mid n \geq 0\right\}$ is an infinite set of preperiodic points, which is again no possible by Northcott theorem (Proposition B.4.2.(b) in [21]).

Observe that the finiteness of the set of $K$-rational trivial points of degree $e>1$ will follow from the same result on the periodic points, which is a conjecture (see for example conjecture 3.15 in [30]).

Question. Are there genus 0 towers (of the form $\mathbf{C}_{\mathcal{F}}$ ) over a number field $K$ having an infinite number of $K$-rational trivial points?

Concerning the trivial points of the genus 0 towers, it is easy to construct examples such that there are infinitely many of them, and towers with only a finite number of them, as shown in the next two examples.

Example. Consider the special case $f(x)=x^{2}$ and $K=\mathbb{Q}$. Then the set of trivial points $\mathbf{C}_{x^{2}}^{(\infty)}(K)_{0}$ of $\mathbf{C}$ in level 0 contains all the $n$-roots of unity for odd $\geq 1$ (and $x=0$ and $\infty$ ), 
since they are defined in a finite extensión of $\mathbb{Q}$ and they are periodic for $f$. It is easy to show that they are all:

$$
\mathbf{C}_{x^{2}}^{(\infty)}(\mathbb{Q})_{0}=\left\{\xi \in \overline{\mathbb{Q}} \mid \exists N \geq 1 \text { such that } \xi^{N}=1\right\} \cup\{0, \infty\} .
$$

Example. Take the genus 0 tower $\mathbf{C}_{\mathcal{F}}$ defined by $\mathcal{F}:=\left\{f_{n}(x)=x^{n+1} \in K(x)\right\}$, where $K$ is any number field. Then the set of trivial points of $\mathbf{C}$ in level 0 is equal to

$$
\mathbf{C}_{\mathcal{F}}^{(\infty)}(K)_{0}=\{0,1, \infty\}
$$

To show these, observe that $\alpha \in K$ is a $K$-rational trivial point if and only if $\alpha$ has a $n$-th root for all $n \geq 1$. But the only such numbers are 0 and 1 in any number field. This last result can be shown using proving first that the absolute logarithmic height of $\alpha$ must be 0 (if $\alpha \neq 0$ ) as in the proof of the theorem 3.3. so $\alpha$ must be a root of unity. But the only root of unity which is and $n$-th root of unity for all $n \geq 1$ is 1 .

\section{Genus 1 Towers}

First of all, observe that, if we have a tower $\mathbf{C}$ of genus 1 curves over a field $K$ such that there is a trivial point $P \in \mathbf{C}(K)_{0}$, we can use this point in order to get an explicit description of the tower.

Lemma 4.1. Consider a tower $\mathbf{C}=\left(\left\{C_{n}\right\}_{n \geq 0},\left\{\varphi_{n}\right\}_{n>1}\right)$ of genus 1 curves over a field $K$, and suppose there is a point $P \in \mathbf{C}(K)_{0}$. Then the tower $\mathbf{C}$ is isomorphic to a tower $\mathcal{E}:=\left(\left\{E_{n}\right\}_{n \geq 0},\left\{\phi_{n}\right\}_{n \geq 1}\right)$ where the $E_{n}$ are elliptic curves and the $\phi_{n}: E_{n} \rightarrow E_{n-1}$ are isogenies.

Proof. Let us fix a point $P_{n} \in C_{n}(K)$ such that $\varphi_{n}\left(P_{n}\right)=P_{n-1}$. Consider the elliptic curve $E_{n}:=\operatorname{Jac}\left(C_{n}\right)$, and the Abel-Jacobi map $\iota_{n}: C_{n} \rightarrow E_{n}$ given by the point $P_{n} \in C_{n}(K)$, which is an isomorphism of curves. The maps $\phi_{n}:=\iota_{n-1} \varphi_{n} \iota_{n}^{-1}$ are non-constant morphisms of curves between $E_{n}$ and $E_{n-1}$ which send the identity point to the identity point. Hence they are isogenies.

Definition. Given an elliptic curve $E$ over a field, denote by $\mathcal{O}:=\operatorname{End}_{K}(E)$ the ring of endomorfisms of $E$ over $K$ as elliptic curve (if $E$ has no complex multiplication, and $K$ has characteristic 0 , then $\mathcal{O} \cong \mathbb{Z}$ ). Given a progression $\mathcal{A}:=\left\{a_{n} \in \mathcal{O} \mid n=0,1, \ldots\right\}$ of elements with degree $>1$, consider the tower of curves $\mathbf{C}_{E, \mathcal{A}}$ defined as $C_{n}:=E$ for all $n \geq 0$ and $\varphi_{n}$ equal to the $a_{n}$ for all $n \geq 1$. The special case that $a_{n}=a$ for all $n$ will be denoted by $\mathbf{C}_{E, a}$.

Corollary 4.2. Let $\mathbf{C}=\left(\left\{C_{n}\right\}_{n \geq 0},\left\{\varphi_{n}\right\}_{n \geq 1}\right)$ be a tower of genus 1 curves over a number field $K$ such that $\mathbf{C}(K)_{0} \neq \emptyset$. Then there is an elliptic curve $E$ defined over $K$ and $a$ progression $\mathcal{A}:=\left\{a_{n}\right\}$ such that the tower $\mathbf{C}_{E, \mathcal{A}}$ is isomorphic to a subtower of $\mathbf{C}$.

Proof. By applying the lemma we are reduced to the case that the $C_{n}$ are elliptic curves and the $\varphi_{n}$ are isogenies. A well-known result of Faltings' (see [15]) implies that there is a finite number of elliptic curves isogenous to a given one. Hence in the set of elliptic curves $C_{n}$, there are infinitely many of them isomorphic to a given elliptic curve $E$. The result is now easily deduced.

Now we can proof the finiteness of the $K$-rational trivial points. 
Corollary 4.3. Let $\mathbf{C}=\left(\left\{C_{n}\right\}_{n \geq 0},\left\{\varphi_{n}\right\}_{n \geq 1}\right)$ be a tower of genus 1 curves over a number field $K$. Then, for all $n \geq 0$ and all $d \geq 1$, the set of $K$-trivial points of $\mathbf{C}$ in the level $n \geq 0$ and degree $d$ is finite.

Proof. Observe that it is sufficient to know the result for an isomorphic subtower. Hence, by using the previous results, we are reduced to the case that $\mathbf{C}=\mathbf{C}_{E, \mathcal{A}}$ for some elliptic curve $E$ and some progression $\mathcal{A}:=\left\{a_{n}\right\}$ of natural numbers, or of elements in a quadratic imaginary order in the CM case, and also the case $n=0$.

Consider a point $P \in \mathbf{C}^{(d)}(K)_{0}$. Let $L / K$ an extension of degree $d$ such that $P \in \mathbf{C}(L)_{0}$. Such an element in $\mathbf{C}(L)_{0}$ is a point $P \in E(L)$ that is divisible by $b_{n}:=a_{0} a_{1} \cdots a_{n}$ for all $n$, hence by its norm to $\mathbb{Z}$. But $E(L)$ is finitely generated, so $P$ must be torsion. Thus $\mathbf{C}(L)_{0} \subset E(L)_{\text {tors }}$, the torsion subgroup, which is finite, which proves the case $d=1$. In general, we get that

$$
\mathbf{C}^{(d)}(K)_{0} \subset \bigcup_{L \subset \bar{K},[L: K] \leq d} E(L)_{\text {tors }}
$$

which is again finite (a fact that can be deduced from Merel's result [27], or by using the results of section 6 due to Frey [17] and the result of Abramovich bounding below the gonality of the modular curves $X_{1}(N)$ [1], or, even, the results in section 8).

On the other hand, it is not true in general that the set of all trivial points of a genus 1 tower is finite, as shown in this example.

Example. The set of all trivial points of the tower $\mathbf{C}_{E, a}$, where $E$ is an elliptic curve defined over a number field $K$ and $a>1$, is equal to the set of torsion points of $E(\bar{K})$ with order prime with $a$ :

$$
\mathbf{C}_{E, N}^{(\infty)}(K)=\{P \in E(\bar{K}) \mid \exists m,[m](P)=0 \text { and }(m, a)=1\} .
$$

Finally, let us mention that there are genus 1 towers without trivial points at all, as shown in the next example.

Example. Let $E$ be an elliptic curve over a number field $K$. Suppose that the Galois cohomology group $H^{1}(K, E)$ contains a divisible element $\psi$, or, even less, an element divisible for all powers of a prime $p$. Now, consider elements $\psi_{n} \in H^{1}(K, E)$ such that $p \psi_{n}=\psi_{n-1}$ and $\psi_{0}=\psi$.

Recall that any element $\xi \in H^{1}(K, E)$ corresponds to a twist $C_{\xi}$ of the curve $E$, that is, a genus 1 curve isomorphic to $E$ over the algebraic clausure of $K$ (and, hence, with jacobian isomorphic to $E$ over $K)$. Moreover, the multiplication-by- $m$ in the group $H^{1}(K, E)$ correspons to a map $\phi_{m \xi}$ between $C_{\xi}$ and $C_{m \xi}$ such that gives the multiplication-by- $m$ map between the corresponding jacobians (see for example [22] for all this facts).

So we have a tower given by the curves $C_{\psi_{n}}$ and the maps $\varphi_{n}:=\phi_{p \psi_{n}}$. Now, the elements $\psi_{n}$ have order divisible by $p^{n}$, hence have index also divisible by $p^{n}$ (see proposition 5 in [22]), which implies that the curves $C_{\psi_{n}}$ do not have rational points in any extension with degree $<p^{n}$. Hence the result.

Now, we only need to show the existence of such elliptic curves $E$. But in fact, showing the existence of one such $E$ over $\mathbb{Q}$ is sufficient. And this is known: take, for example, any elliptic curve $E$ over $\mathbb{Q}$ with finite number of $\mathbb{Q}$-rational points (see for example theorem D in [9]). 


\section{Finiteness of trivial points And REDUCtion}

Now we are going to consider towers with genus $\infty$, or, equivalently, towers such that there is a curve $C_{n}$ with genus $>1$. In this case, and when $K$ is a number field, the finitness of the $K$-rational trivial points is clear, since, by Faltings' theorem [15], the number of points in $C_{n}(K)$ is finite. So we are mainly interested in the whole trivial points. Next example will show that there are towers with genus infinite and an infinite number of trivial points.

Example. For $n \geq 0$, let $C_{n}$ be the smooth hyperelliptic curve defined over $\mathbb{Q}$ by the hyperelliptic equation $y^{2}=x^{2^{n}}-1$, and consider the degree two maps $\varphi_{n}$ defined in the affine part by $\varphi_{n}(x, y)=\left(x^{2}, y\right)$.

Now, take $\xi \in \overline{\mathbb{Q}}$ a root of unity of odd degree, so there exists an odd $N \geq 1$ such that $\xi^{N}=1$. Consider the field $K_{\xi}$ generated by $\sqrt{\xi^{i}-1}$, for $i=1, \ldots, N-1$; it is a finite extension of $\mathbb{Q}$ and, clearly, $(\xi, \sqrt{\xi-1}) \in C_{0}\left(K_{\xi}\right)$ is a $K_{\xi}$-rational trivial point of the tower.

So, the trivial points of the tower include all the points of this form, which are infinite.

Observe that hyperelliptic curves have an infinite number of points of degree 2, a result that generalizes to points of degree $d$ and gonality $d$.

Question. For $d>1$, are there towers with infinite genus over a number field $K$ having an infinite number of trivial points of degree $d$ ?

We will see in the next section that the answer of the question is no when the gonality of the tower is infinite. But before we will give a criterion for a tower to have a finite number of trivial points.

Definition. Let $\mathbf{C}$ be a tower of curves over a number field $K$ and a ring of integers $\mathcal{O}$ (a Dedekind domain, not a field, and with field of fractions $K$ ). By a proper model of $\mathbf{C}$ over $\mathcal{O}$ we mean a collection of proper models $\mathcal{C}_{n}$ of $C_{n}$ and morphisms $\varphi_{n, \mathcal{O}}: \mathcal{C}_{n} \rightarrow \mathcal{C}_{n-1}$ such that the generic fiber is $\varphi_{n, \mathcal{O}} \otimes_{\mathcal{O}} K=\varphi_{n}$. We will denote by $\widetilde{\varphi_{n, \wp}}: \mathcal{C}_{n, \wp} \rightarrow \mathcal{C}_{n-1, \wp}$ the reduction of the morphism modulo some prime $\wp$ of $\mathcal{O}$ (and we will suppress the $\wp$ in the notation if it is clear from the context).

Observe that for any prime $\wp$ of $\mathcal{O}$ we have a reduction $\operatorname{map} \operatorname{red}_{\wp}: \mathcal{C}_{n}(\mathcal{O}) \rightarrow \mathcal{C}_{n, \wp}\left(k_{\wp}\right)$, where $k_{\wp}$ is the residue field $\mathcal{O} / \wp$. For any $P \in C_{n}(K)=\mathcal{C}_{n}(\mathcal{O})$, we have $\operatorname{red}_{\wp}\left(\varphi_{n}(P)\right)=$ $\widetilde{\varphi_{n}}\left(\operatorname{red}_{\wp}(P)\right)$.

Theorem 5.1. Let $\mathbf{C}$ be a tower of curves over a number field $K$ such that $\Omega:=\mathbf{C}(K)_{n}$ is finite for some $n \geq 0$. Fix a proper model of $\mathbf{C}$ over a ring of integers $\mathcal{O}$ of $K$, and suppose that for any prime $\wp$ of $\mathcal{O}$ outside a finite number of primes, there exists $m:=m_{\wp} \geq n$ such that $C_{m}\left(k_{\wp}\right)=\operatorname{red}_{\wp}\left(\varphi_{m, n}^{-1}(\Omega)\right)$, where $k_{\wp}$ is the residue field modulo $\wp$ and red $d_{\wp}$ is the reduction map. Then $\Omega$ is the set of all trivial points $\mathbf{C}^{(\infty)}(K)_{n}$ of $\mathbf{C}$, and there is a finite number.

Proof. Let $L / K$ be a finite extension of $K$, and let $\mathcal{O}_{L}$ be the ring of integers of $L$. We are going to show that the set of $L$-rational trivial points $\mathbf{C}(L)_{n}$ is equal to $\Omega$. Suppose in the contrary that there is a point $P \in\left(\mathbf{C}(L)_{n} \backslash \Omega\right)$.

Consider a prime ideal $\wp_{L}$ of $\mathcal{O}_{L}$ such that there is a prime ideal $\wp$ of $K$ divisible by $\wp_{L}$ and with equal residue fields $k_{\wp_{L}}=k_{\wp}$ (there are an infinite number of them). First, we show 
that then $\operatorname{red}_{\wp_{L}}(P) \in \operatorname{red}_{\wp}(\Omega)$. Take $m:=m_{\wp}$ and $P_{m} \in C_{m}(L)$ such that $\varphi_{m, n}\left(P_{m}\right)=P$. By hypothesis, $\operatorname{red}_{\wp_{L}}\left(P_{m}\right) \in \operatorname{red}_{\wp}\left(\varphi_{m, n}^{-1}(\Omega)\right)$. Hence

$$
\widetilde{\varphi_{m, n}}\left(\operatorname{red}_{\wp_{L}}\left(P_{m}\right)\right)=\operatorname{red}_{\wp_{L}}\left(\varphi_{m, n}\left(P_{m}\right)\right)=\operatorname{red}_{\wp_{L}}(P) \in \widetilde{\varphi_{m, n}}\left(\operatorname{red}_{\wp}\left(\varphi_{m, n}^{-1}(\Omega)\right)\right)=\operatorname{red}_{\wp}(\Omega) .
$$

So, we have an infinite number of primes $\wp_{L}$ of $\mathcal{O}_{L}$ such that $\operatorname{red}_{\wp_{L}}(P) \in \operatorname{red}_{\wp_{L}}(\Omega)$. Since the set $\Omega$ is finite, there should exists a point $Q \in \Omega$ and an infinite number of primes $\wp_{L}$ of $\mathcal{O}_{L}$ such that $\operatorname{red}_{\wp_{L}}(P)=\operatorname{red}_{\wp_{L}}(Q)$. But, given a proper curve $\mathcal{C}$ over a Dedekind domain $\mathcal{O}$ and points $P \neq Q \in \mathcal{C}(\mathcal{O})$, the number of primes $\wp$ of $\mathcal{O}$ such that $\operatorname{red}_{\wp}(P)=\operatorname{red}_{\wp}(Q)$ is finite. Hence $P=Q$.

Corollary 5.2. Suppose that the tower $\mathbf{C}$ has a model with good reduction outside a finite number of primes $S$ of $\mathcal{O}$. For any prime $\wp$ of good reduction of $\mathbf{C}$, consider the tower of curves $\mathbf{C}_{\wp}=\left(\left\{\mathrm{C}_{n, \wp}\right\},\left\{\widetilde{\varphi_{n, \wp}}\right\}\right)$, reduction modulo $\wp$ of the model. Suppose that there exists $n$ such that for any prime $\wp$ outside a finite set containing $S, \operatorname{red}_{\wp}\left(\mathbf{C}(K)_{n}\right)=\mathbf{C}_{\wp}\left(k_{\wp}\right)_{n}$. Then $\mathbf{C}^{(\infty)}(K)_{n}=\mathbf{C}(K)_{n}$.

One can use this result to show that for some modular towers the trivial points are the cusps. The next example is a generalization of the curves $X_{1}\left(p^{n}\right)$ to higher dimensional abelian varieties.

Example. Let $K$ be a number field and $U / k$ a smooth geometrically connected algebraic curve over $K$. Let $\mathcal{A} \rightarrow U$ be an abelian scheme of dimension $g \geq 1$, defined over $K$. Given a prime number $p$ and a $n \geq 1$, consider the possibly disconnected curve $\mathcal{A}\left[p^{n}\right] \rightarrow U$ over $U$, and let $U_{n}$ be a geometrically connected component of it, such that the multiplication-by- $p$ maps $\mathcal{A}\left[p^{n}\right] \rightarrow \mathcal{A}\left[p^{n-1}\right]$ give maps $\phi_{n}: U_{n} \rightarrow U_{n-1}$ for all $n \geq 2$. Let $C_{n}$ be the desingularization of some projectivization of $U_{n}$, together with the natural maps $\varphi_{n}: U_{n} \rightarrow U_{n-1}$. Since the $U_{n}$ are smooth over $U$, the points $U_{n}(L)$ can be seen inside $C_{n}(L)$, for any extension $L / K$. Then the trivial points $\mathbf{C}^{(\infty)}(K)_{n}$ of the tower $\mathbf{C}=\left(\left\{C_{n}\right\}_{n \geq 0},\left\{\varphi_{n}\right\}_{n \geq 1}\right)$ at level $n$ are contained in $C_{n}(\bar{K}) \backslash U_{n}(\bar{K})$, which is well known to be finite.

This result can be shown by observing that the points of $s \in U_{n}(L)$ correspond to a special fiber $\mathcal{A}_{s}$, which is an abelian variety over $L$, together with a point $P \in \mathcal{A}_{s}(L)$ of order exactly $p^{n}$. This point $s$ is a trivial point if and only if for all $m \geq 1$, there exists a point $Q \in \mathcal{A}_{s}(L)$ such that $\left[p^{m}\right] Q=P$; thus, $Q$ has exact order $p^{n+m}$. Since the cardinal of the group of torsion points of an abelian variety over a number field is finite (a fact that can be proved by reducing modulo some primes $\ell$ ), there is no such a $Q$ for $m \gg 1$. So there is no trivial point inside $U_{n}$.

Another way to show this result is by reducing modulo some prime $\wp$ of $K$, such that the map $\mathcal{A} \rightarrow U$ has good reduction, and does not divide $p$ (all primes except a finite number of them verify these conditions). Then the tower $U_{n}$ has good reduction at such a prime. The assertion is deduced from the fact that the cardinal of the group of (torsion) points of an abelian variety over a finite field is finite, which is trivial, and then applying the corollary above.

\section{Genus $\infty$ Towers, trivial points And Gonality}

Recall that the gonality $\gamma_{K}(C)$ of a curve $C$ over a field $K$ is the minimum $m$ such that there exists a morphism $\phi: C \rightarrow \mathbb{P}^{1}$ of degree $m$ defined over $K$. For example, hyperelliptic 
curves have gonality 2 . In the next proposition we recall some properties of the gonality (see [29] for the proofs).

Proposition 6.1. Let $K$ be any field, and let $C$ be an smooth and projective curve with genus $g>1$ y gonality $\gamma_{K}(C)$. Then

(1) $\gamma_{K}(C) \leq 2 g-2$.

(2) If $C(K) \neq \emptyset$, then $\gamma_{K}(C) \leq g$.

(3) If $K=\bar{K}$ is algebraically closed, then $\gamma_{K}(C) \leq\left\lfloor\frac{g+3}{2}\right\rfloor$.

(4) If $L / K$ is a field extension, then $\gamma_{K}(C) \geq \gamma_{L}(C)$.

(5) If $K$ is a perfect field, $L / K$ is an algebraic field extension, $\gamma_{L}(C)>2$ and $C(K) \neq \emptyset$ then $\gamma_{K}(C) \leq\left(\gamma_{L}(C)-1\right)^{2}$.

(6) If $f: C \rightarrow C^{\prime}$ is a non-constant $K$-morphism then $\gamma_{K}(C) \leq \operatorname{deg}(f) \gamma_{K}\left(C^{\prime}\right)$ and $\gamma_{K}\left(C^{\prime}\right) \leq \gamma_{K}(C)$.

The main tool we will use to relate the gonality with the finiteness of trivial points is the following criterion of Frey [17], proved also by Abramovich in his thesis, which is an application of the main result of Faltings in [16].

Theorem 6.2 (Frey). Let $C$ a curve over a number field $K$, with gonality $\gamma>2$ over $K$. Fix an algebraic closure $\bar{K}$ of $K$ and consider the points of degree d of $C$,

$$
C^{d}(K):=\bigcup_{[L: K] \leq d} C(L) \subset C(\bar{K})
$$

where the union is over all the finite extensions of $K$ inside $\bar{K}$ of degree $\leq d$. Suppose that $2 d<\gamma$. Then $C^{d}(K)$ is finite.

Using this criterion we can show the following result.

Corollary 6.3. Let $\mathbf{C}$ be a tower with infinite gonality. Then, for all $d \geq 1$ and $n \geq 0$, the set of $K$-trivial points of $\mathbf{C}$ in the level $n \geq 0$ and degree $d$ is finite.

Moreover, for all $d \geq 1$ there exists a constant $n_{d}$ (depending on the tower $\mathbf{C}$ ) such that, for any extension $L / K$ of degree $\leq d$, and for any $n \geq n_{d}, C_{n}(L) \subset \mathbf{C}^{(d)}(K)_{n}$.

Corollary 6.4. If the set $\mathbf{C}^{(\infty)}(K)_{n}$ is finite for some $n$, and $\gamma(\mathbf{C})=\infty$, then for any extension $L / K$ of degree $\leq d$ and for any $n \geq n_{d}, C_{n}(L) \subset \mathbf{C}^{(\infty)}(K)_{n}$.

Question. Are there towers with infinite gonality over a number field $K$ having an infinite number of trivial points?

\section{Gonality in the COMPlete interseCtion CASE}

Definition. For any $n \geq 1$, let $f_{n}\left(X_{0}, X_{1}, \ldots, X_{n+1}\right)$ be a homogeneous polynomial of degree $d_{n}>1$. Consider the curves $C_{n-1} \subset \mathbb{P}^{n+1}$ defined as the zero set of the polynomials $f_{1}, f_{2}, \ldots, f_{n}$. We have a natural map $\varphi_{n}: C_{n} \rightarrow C_{n-1}$ given by forgetting the last coordinate: $\varphi_{n}\left(\left[x_{0}: x_{1}: \cdots: x_{n+2}\right]\right)=\left[x_{0}: x_{1}: \cdots: x_{n+1}\right]$. If the polynomials $f_{n}$ are sufficiently general, then the curves $C_{n}$ are smooth and complete intersection, and we get a tower of curves. We call these type of towers complete intersection towers.

The main result of this section is that the complete intersection towers have infinite gonality. The main tool is the following theorem by Lazardsfeld (see Exercise 4.12. in [23]), 
which is a generalization of the well-known fact that a planar curve (so given by an smooth projective model inside $\mathbb{P}^{2}$ ) has gonality equal to the degree of the model minus 1 if the curve has a rational point.

Theorem 7.1 (Lazarsfeld). Let $C \subset \mathbb{P}^{n}$ be a smooth complete intersection of hypersurfaces of degrees $2 \leq a_{1} \leq a_{2} \leq \cdots \leq a_{r-1}$ over $\mathbb{C}$. Then $\gamma(C) \geq\left(a_{1}-1\right) a_{2} \cdots a_{r-1}$.

Corollary 7.2. Any complete intersection tower of curves has infinite gonality over any characteristic zero field.

Proof. Let $a_{1}:=\min d_{n}$ and let $n_{0}$ such that $d_{n_{0}}=a_{1}$. Then the gonality of the curves $C_{n-1}$ for $n \geq n_{0}$ will be bounded below by

$$
\gamma\left(C_{n-1}\right) \geq\left(1-\frac{1}{a_{1}}\right)\left(\prod_{i=1}^{n} d_{n}\right)
$$

hence its limit goes to infinite.

Observe that, if the degree $d_{1}$ is the minimum of all the $d_{n}$, and $C_{0}(K) \neq \emptyset$, then the gonality of $C_{n}$ over $K$ is in fact equal to $\left(d_{1}-1\right)\left(\prod_{i=2}^{n} d_{n}\right)$, since the map $\varphi_{n, 1}: C_{n} \rightarrow C_{0}$ has degree $\left(\prod_{i=2}^{n} d_{n}\right)$, which composed with the map of degree $d_{1}-1$ from $C_{0}$ to $\mathbb{P}^{1}$ given by the rational point has the desired degree.

Example. Fix an homogeneous irreducible polynomial $f\left(X_{0}, X_{1}, X_{2}\right)$ of degree $d>1$ and defined over a number field. Suppose that the curve projective $C_{0} \subset \mathbb{P}^{2}$ defined as the zero set of the polynomials $f$ is non singular and geometrically connected. Consider now the complete intersection tower $\mathbf{C}_{f}$ of curves $C_{n-1} \subset \mathbb{P}^{n+1}$ defined as the zero set of the polynomials $f_{1}:=f, f_{2}:=f\left(X_{1}, X_{2}, X_{3}\right), \ldots, f_{n}:=f\left(X_{n-2}, X_{n-1}, X_{n}\right)$. Then the curve $C_{n}$ has gonality $\geq d^{n}-d^{n-1}$, so the tower has infinite gonality, with equality exactly when $C_{0}(K) \neq \emptyset$.

Hence, if $K$ is a number field, and by Corollary 6.3, we get that the curves $C_{n}$ have only the trivial points over a finite extension $L / K$ for $n$ large enough, depending only on the tower and the degree $[L: K]$.

And, in case we know the set of trivial points is finite and computable (for example, by theorem 5.1, we get that the points of the curves $C_{n}$ over a finite extension $L / K$ for $n$ large enough, depending only on $\mathbf{C}$ and the degree $[L: K]$, by Corollary 6.4 .

One can also use the theorem for other type of towers of curves, a generalization of the $p^{n}$ Fermat tower.

Example. Fix an homogeneous irreducible polynomial $f_{0}\left(X_{0}, X_{1}, X_{2}\right)$ of degree $d>1$ and defined over a number field, and a progression $\left\{a_{n}\right\}_{n \geq 1}$ of integers $a_{n} \geq 2$. Suppose that the curve projective $C_{0} \subset \mathbb{P}^{2}$ defined as the zero set of the polynomials $f_{0}$ is non singular and geometrically connected. Consider now the complete intersection tower $\mathbf{C}$ of curves $C_{n} \subset \mathbb{P}^{2}$ defined as the zero set of the polynomial $f_{n}:=f_{n-1}\left(X_{0}^{a_{n}}, X_{1}^{a_{n}}, X_{2}^{a_{n}}\right)$, and the maps $\varphi_{n}$ given by $\varphi_{n}\left(X_{0}, X_{1}, X_{2}\right)=\left[X_{0}^{a_{n}}: X_{1}^{a_{n}}: X_{2}^{a_{n}}\right]$. Then the curves $C_{n}$ are planar curves of degree $d a_{1} a_{2} \cdots a_{n}$, and hence with gonality $\gamma\left(C_{n}\right) \geq d a_{1} a_{2} \cdots a_{n}-1 \geq 2^{n}-1$.

\section{Gonality And Reduction}

The following proposition was shown in [31], proposition 5, and it will allow us to bound by below the gonality by just counting points "modulo p". 
Proposition 8.1. Let $C$ be a curve over a number field, and let $\wp$ be a prime of good reduction of the curve, with residue field $k_{\wp}$. Denote by $C^{\prime}$ the reduction of the curve $C$ modulo $\wp$. Then the gonality $\gamma(C)$ of $C$ satisfies that

$$
\gamma\left(C_{K}\right) \geq \gamma\left(C_{k_{\wp}}^{\prime}\right) \geq \frac{\sharp C^{\prime}\left(k_{\wp}\right)}{\sharp k_{\wp}+1} .
$$

Observe that the proposition has two parts: first, that the gonality does not increase under reduction modulo a prime. Second, that the gonality over a finite field is bounded below by the number of points.

Definition. Let $K$ be a number field and let $\mathbf{C}$ be a tower of curves over $K$. We will say that a prime $\wp$ of the ring of integers $\mathcal{O}$ of $K$ is a prime of good reduction of the tower if there exists a proper model of $\mathbf{C}$ over $\mathcal{O}_{(\wp)}$, the localization of $\mathcal{O}$ at $\wp$, such that $\mathcal{C}_{n, \wp}$ are smooth and projective curves, and the maps $\widetilde{\varphi_{n, \wp}}$ are non constant.

Corollary 8.2. Let $\mathbf{C}=\left(\left\{C_{n}\right\}_{n \geq 0},\left\{\varphi_{n}\right\}_{n \geq 1}\right)$ be a tower of curves over a number field $K$. Suppose that there exists a prime $\wp$ of good reduction of the tower, and suppose that

$$
\lim _{n \rightarrow \infty} \sharp \mathfrak{C}_{n, \wp}\left(k_{\wp}\right)=+\infty \text {. }
$$

Then the tower $\mathbf{C}$ has infinite gonality.

Proof. Using the proposition 8.1 one gets that

$$
\gamma\left(C_{n}\right) \geq \gamma\left(\mathfrak{C}_{n, \wp}\right) \geq \frac{\sharp \mathcal{C}_{n, \wp}\left(k_{\wp}\right)}{\sharp k_{\wp}+1},
$$

hence the result.

\section{Gonality and Cayley-Schreier graphs}

In this section we are going to follow the ideas originating in the work of Zograf [32] and Abramovich [1], and developed in a recent paper of Ellenberg, Hall and Kowalski [14. The idea is to show that certain étale towers of (possibly affine) curves have infinite gonality if the associated Cayley-Schreier graphs form an expanding family (or, more generally, verify some growing condition in the first non-trivial eigenvalue of the combinatorial laplacian operator).

Suppose that we have a tower of curves $\mathbf{C}$ defined over a number field such that the maps $\varphi_{n, 0}$ are étale (i.e. non-ramified) outside a fixed Zarisky closed set $Z$. So, we have open subsets $U_{i}=C_{i} \backslash Z$ of $C_{i}$, together with maps $\varphi_{n}: U_{n} \rightarrow U_{n-1}$, which are étale, and the original tower $\mathbf{C}$ is obtained projectivizing the curves $U_{i}$ (the case $U_{i}=C_{i}$ is also considered).

Fix, for all $i \geq 0$, a point $x_{i}$ in $U_{i}(\bar{K})$ such that $\varphi_{i}\left(x_{i}\right)=x_{i-1}$, and a generating set $S$ of the topological fundamental group $G:=\pi_{1}\left(U_{0 \mathbb{C}}, x_{0}\right)$. Consider the Cayley-Schreier graphs $\Gamma_{i}=C\left(N_{i}, S\right)$ associated to the finite quotient sets

$$
N_{i}:=G / H_{i}=\pi_{1}\left(U_{0 \mathbb{C}}, x_{0}\right) / \pi_{1}\left(U_{i \mathbb{C}}, x_{i}\right) .
$$

Recall that the graphs $\Gamma_{i}=C\left(G / H_{i}, S\right)$ have vertex set $V\left(\Gamma_{i}\right)=G / H_{i}$, and with (possibly multiple) edges from vertex $x H_{i}$ to vertex $s x H_{i}$ for all $s \in S$; hence, they are $r$-regular graphs for $r=|S|$.

Define the combinatorial Laplacian operator of a $r$-regular graph $\Gamma$ as $r I d-A\left(\Gamma_{i}\right)$, where $A(\Gamma)$ is the adjacency matrix of $\Gamma$. We compute the eigenvalues of $\Gamma$, which are positive real numbers, and let $\lambda_{1}(G)$ to be the smallest non-zero of them. 
Observe that there are maps of graphs $\Gamma_{i} \rightarrow \Gamma_{i-1}$ for all $i \geq 1$, and that such maps are unramified: the preimatge of any vertex is formed always by $k$ vertices, where $k$, the degree of the map, is fixed.

Definition. A tower of graphs is a couple $\left(\left\{\Gamma_{i}\right\},\left\{\phi_{i}\right\}\right)$ where $\Gamma_{i}$ are graphs for any $i \geq 0$ and $\phi_{i}: \Gamma_{i} \rightarrow \Gamma_{i-1}$ are surjective maps of graphs. We say that the tower is unramified if all the maps $\phi_{i}$ for $i \geq 1$ are unramified.

Following ideas from the paper [14], we will bound the gonality of the curves $C_{i}$ by imposing some condition on the first non-zero eigenvalue $\lambda_{1}\left(\Gamma_{i}\right)$ of the combinatorial laplacian operator on $\Gamma_{i}$.

Theorem 9.1. Let $\mathbf{C}=\left(\left\{C_{n}\right\},\left\{\varphi_{n}\right\}\right)$ be a tower of curves defined over $\mathbb{C}$ such that the maps $\varphi_{n, 0}$ are étale outside a fixed Zarisky closed set $Z$, and with the genus $g\left(C_{0}\right)>$ 1. Consider the open subsets $U_{i}=C_{i} \backslash Z$ of $C_{i}$, together with maps $\varphi_{n}: U_{n} \rightarrow U_{n-1}$ and points $x_{i}$ in $U_{i}(\bar{K})$ such that $\varphi_{i}\left(x_{i}\right)=x_{i-1}$. Let $\left\{\Gamma_{i}, \phi_{i}\right\}$ be the unramified tower of Cayley-Schreier graphs $C\left(\pi_{1}\left(U_{0 \mathbb{C}}, x_{0}\right) / \pi_{1}\left(U_{i \mathbb{C}}, x_{i}\right), S\right)$. Suppose that $\lim _{i \rightarrow \infty} \lambda_{1}\left(\Gamma_{i}\right)\left|V\left(\Gamma_{i}\right)\right|=$ $\infty$. Then $\lim _{i \rightarrow \infty} \gamma\left(C_{i}\right)=\infty$.

Proof. First of all, observe that, by the Hurwitz's formula

$$
g\left(C_{i}\right)-1 \geq \operatorname{deg}\left(\varphi_{i, 0}\right)\left(g\left(C_{0}\right)-1\right) \geq \operatorname{deg}\left(\varphi_{i, 0}\right)
$$

(where we have equality exactly if $\phi_{i, 0}$ are unramified). Now, the degree of $\varphi_{i, 0}$ is exactly equal to the index of $\pi_{1}\left(U_{i \mathbb{C}}, x_{i}\right)$ inside $\pi_{1}\left(U_{0 \mathbb{C}}, x_{0}\right)$, which is equal to the number of vertices of $\Gamma_{i}$. So we get that $g\left(C_{i}\right)-1 \geq\left|V\left(\Gamma_{i}\right)\right|$.

Now, we will find a formula relating the $\lambda_{1}\left(\Gamma_{i}\right)$ to the gonality of $C_{i}$ and $g\left(C_{i}\right)$. We will follow the proof of Theorem 8 (b) in [14], and we will only sketch the proof. Since the genus of $C_{i}$ is $>1$ for all $i$, we can write $U_{i}$ as $G_{i} \backslash \mathbb{H}$ for some discrete subgroup of $P S L_{2}(\mathbb{R})$. The hyperbolic area $\mu_{i}\left(U_{i}\right)$ is then finite and the Poincaré metric induces a Laplacian operator on the $L^{2}$-space. Following Li and Yau [24], and Abramovich [1], one has that

$$
\gamma\left(C_{i}\right) \geq \frac{1}{8 \pi} \lambda_{1}\left(U_{i}\right) \mu\left(U_{i}\right)
$$

where $\lambda_{1}\left(U_{i}\right)$ is the first non-trivial eigenvalue of the laplacian operator -div(grad).

Now, using the Gauss-Bonnet theorem, one gets

$$
\mu\left(U_{i}\right)=-2 \pi \chi\left(U_{i}\right) \geq-2 \pi \chi\left(C_{i}\right)=-4 \pi\left(1-g\left(C_{i}\right)\right) .
$$

Using the comparison principle of Brooks [7] and Burger [8], one gets that there exists a constant $c>0$, depending only on $U_{0}$ and on $S$, such that

$$
\lambda_{1}\left(U_{i}\right) \geq c \lambda_{1}\left(\Gamma_{i}\right) .
$$

Hence, combining all the results, that

$$
\gamma\left(U_{i}\right) \geq 2 c \lambda_{1}\left(\Gamma_{i}\right)\left(g\left(C_{i}\right)-1\right) \geq 2 c \lambda_{1}\left(\Gamma_{i}\right)\left|V\left(\Gamma_{i}\right)\right|
$$

and hence the result.

We say that a family of graphs is an expander if $\lim _{i \rightarrow \infty}\left|\Gamma_{i}\right|=\infty$ and $\lambda_{1}\left(\Gamma_{i}\right) \geq c$ for some constant $c$. We will say that it is esperantist if there exists some constant $A \geq 0$ such that

$$
\lambda_{1}\left(\Gamma_{i}\right) \geq \frac{c}{\left(\log \left(2\left|\Gamma_{i}\right|\right)\right)^{A}} .
$$


Observe that, if our family (in fact, tower) of Cayley-Schreier graphs $\left\{\Gamma_{i}\right\}$ is an expander (or it is esperantist), then they verify a fortiori the hypothesis of the theorem. Hence, the following constructions give towers of curves $\mathbf{C}$ defined over a number field $K$ with infinite gonality. Consider $U_{0}$ a smooth geometrically connected algebraic curve over a number field $K$, and suppose we have an epimorfism of groups $p: \pi_{1}\left(U_{0}(\mathbb{C}), x_{0}\right) \rightarrow G$, where the group $G$ is one of the cases below. Take finite-index subgroups $H_{n}$ of $G$ such that $H_{n} \varsubsetneqq H_{n-1}$ and $H_{0}=G$, and consider the étale coverings $U_{n} \rightarrow U_{0}$ associated to the subgroups $p^{-1}(H)$. Finally, consider the projectivizations and desingularizations $C_{n}$ of these curves $U_{n}$.

(1) If $G$ is a finite-index subgroup in $\mathbf{G}(\mathbb{Q}) \cap \mathrm{GL}_{m}(\mathbb{Z})$, where $\mathbf{G} \subset \mathrm{GL}_{m}$ is a semisimple algebraic subgroup, defined over $\mathbb{Q}$, and $G$ has real rank at least 2 (for example, $G$ can be a finite-index subgroup of $\mathrm{SL}_{n}(\mathbb{Z}), n>3$, or of $\mathrm{Sp}_{2 g}(\mathbb{Z}), g>2$ ) and $S$ is an arbitrary finite set of generators of $G$, and $H_{n}$ arbitrary normal subgroups (by property $(\mathrm{T})$ of Kazhdan, see [4]).

(2) If $G$ is a subgroup of $\mathrm{SL}_{n}(\mathbb{Z})$ which is Zarisky-dense in $\mathrm{SL}_{d}$, for $d>1, S$ is an arbitrary finite set of generators of $G, p$ is a prime number sufficiently large (depending on $G$ ) and $H_{n}=p^{n} \mathrm{SL}_{d}(\mathbb{Z})$, so $G / H_{n} \cong \mathrm{SL}_{n}\left(\mathbb{Z} / p^{n} \mathbb{Z}\right)$, by [5] and [6].

(3) If $G$ is any Zarisky dense subgroup of $\mathbf{G} / \mathbb{Z}_{p}$, an arbitrary split semisimple algebraic group, and we consider the tower of Cayley graphs of $\mathbf{G}\left(\mathbb{Z} / p^{n} \mathbb{Z}\right)$ with respect to any symmetric set of generators, by the results of Dinai [12, [13] concerning the diameter of this graphs: the diameter is less than $c \log \left(\left|\mathbf{G}\left(\mathbb{Z} / p^{n} \mathbb{Z}\right)\right|\right)^{d}$, for some constants $c$ and $d$.

To show some of these cases, one needs to know that there is a relation between the first non-trivial eigenvalue of the combinatorial laplacian operator and the diameter (longest shortest path between any to pair of vertices) for any (regular) graph $\Gamma$. For example, Diaconis and Saloff-Coste [11] showed that

$$
\lambda_{1}(C(G, S)) \geq \frac{1}{|S| \operatorname{diam}(C(G, S))^{2}},
$$

if $C(G, S)$ is a Cayley Graph associated to a finite group $G$ with symmetric set of generators $S$. Hence, if e have normal subgroups $H_{i} \unlhd G$ inside a group $G$, with $H_{i} \subseteq H_{i-1}$, the hypothesis of the theorem is verified if

$$
\lim _{i \rightarrow \infty} \frac{\left|G / H_{i}\right|}{\operatorname{diam}\left(C\left(G / H_{i}, S\right)\right)^{2}}=+\infty
$$

On the other hand, it is easy to construct towers of curves not verifying the growing condition on the first non-trivial eigenvalue of the combinatorial laplacian operator (and having bounded gonality), as in the following trivial example.

Example. Consider the tower of curves $\mathbf{C}_{x^{2}}$, so with $C_{n}=\mathbb{P}^{1}$ and maps given by $\varphi_{n}(x)=x^{2}$. Of course this tower has no infinite gonality. These maps $\varphi_{n}$ are unramified outside $x=0$ and $\infty$, so they give unramified selfmaps of $U_{i}=\mathbb{G}_{m}=\mathbb{P}^{1} \backslash\{0, \infty\}$. Choose, for example, $x_{n}:=1 \in C_{n}(\mathbb{Q})$. Then one has that $\pi_{1}\left(U_{i \mathbb{C}}, x_{i}\right) \cong \mathbb{Z}$, and for the set $S:=\{1,-1\}$, we get that the Cayley-Schreier graphs

$$
C\left(\pi_{1}\left(U_{0 \mathbb{C}}, x_{0}\right) / \pi_{1}\left(U_{i \mathbb{C}}, x_{i}\right), S\right)=C\left(\mathbb{Z} / 2^{n} \mathbb{Z},\{ \pm 1\}\right)=\Gamma_{2^{n}}
$$

where $\Gamma_{n}$ denotes the cycle graph form by a cycle with $n$ vertices and $n$ edges. It is well known that the eigenvalues of the combinatorial laplacian operator for these cycle graphs 
are $\lambda_{k}\left(\Gamma_{n}\right):=2-2 \cos (2 k \pi / n)$ for $k=0, \ldots, n-1$. Hence,

$$
\lim _{i \rightarrow \infty} \lambda_{1}\left(\Gamma_{2^{i}}\right)\left|V\left(\Gamma_{2^{i}}\right)\right|=\lim _{i \rightarrow \infty} 2^{i}\left(2-2 \cos \left(\frac{\pi}{2^{i-1}}\right)\right)=0 .
$$

Observe that there is another completely different relation of the gonality with graph theory, developed by Baker and Norine [3] and specially by Baker in [2].

Suppose $K$ is a field complete with respect to a discrete valuation, and let $C$ be a curve over $K$ having a regular semistable model over the ring of integers of $K$. Consider $\Gamma$ the dual graph of the reduction of $X$, where the vertexes are the irreducible components, and the edges correspond to the intersection points. Then, there is a notion of gonality for a finite graph and the gonality of $C$ is bounded below by the gonality of $\Gamma$ (see Corollary 3.2 in [2]). Using this result it is not difficult to construct towers of (Mumford) curves over $\mathbb{Q}_{p}$ having infinite gonality.

All these results are also related to tropicalizations of algebraic curves, and how to bound the gonality from the tropicalization, a subject that we will explore in the future.

\section{REFERENCES}

[1] Abramovich. D. A linear lower bound on the gonality of modular curves. International Math. Res. Notices 20 (1996), 1005-1011.

[2] Baker, M. Specialization of Linear Systems from Curves to Graphs, Algebra and Number Theory 2, no. 6 (2008), 613-653.

[3] Baker, M., Norine, S. Riemann-Roch and Abel-Jacobi Theory on a Finite Graph, Advances in Mathematics 215 (2007), 766-788.

[4] BekKa, B., De la Harpe, P., Valette, A., Kazhdan's property (T), New Mathematical Monographs, 11, Cambridge University Press,(2008).

[5] Bourgain, J., Gamburd, A. Expansion and random walks in $S L_{d}\left(\mathbb{Z} / p^{n} \mathbb{Z}\right)$ :I. J. Eur. Math. Soc. 10, 987-1011 (2008)

[6] Bourgain, J., Gamburd, A. Expansion and random walks in $S L_{d}\left(\mathbb{Z} / p^{n} \mathbb{Z}\right)$ : II. J. Eur. Math. Soc. 11, 1057-1103 (2009)

[7] Brooks, R. On the angles between certain arithmetically defined subspaces of $\mathbb{C}^{n}$. Annales Inst. Fourier 37 (1987), 175-185.

[8] Burger, M. Estimations de petites valeurs propres du laplacien d'un revêtement de variétés riemanniennes compactes. C.R. Acad. Sc. Paris 302 (1986), 191-194.

[9] Çiperiani,M., Stix, J. Weil-Châtelet divisible elements in Tate-Shafarevich groups, arXiv:1106.4255.

[10] Cadoret, A., Tamagawa, A. Uniform boundedness of p-primary torsion of abelian schemes, to apper in Invent. Math.

[11] Diaconis, P., Saloff-Coste, L., Comparison Techniques for Random Walk on Finite Groups, Ann. Probab. 21, no. 4 (1993), 2131-2156.

[12] Dinai, O. Poly-log diameter bounds for some families of finite groups, Proc. Amer. Math. Soc. 134 (2006), 3137-3142.

[13] DinAI, O. Rapid generation and Diameters of Chevalley groups over local rings, preprint.

[14] Ellenberg, J., Hall, C., Kowalski, E. Expander graphs, gonality and variation of Galois representations, arxiv: 1008.3675 .

[15] Faltings, G. Endlichkeitssätze für abelsche Variatäten über Zahlkörpern. Invent. math. 73 (1983), 349-366.

[16] Faltings, G. Diophantine approximation on abelian varieties. Annals of Math. 133 (1991), 549-576.

[17] Frey, G. Curves with infinitely many points of fixed degree. Israel J. Math. 85 (1994), no. 1-3, 79-83.

[18] FRIED, M. D. Introduction to modular towers: generalizing dihedral group-modular curve connections, in Recent developments in the inverse Galois problem (Seattle, WA, 1993), 111-171, Contemp. Math., 186, Amer. Math. Soc., Providence, RI, 1995. 
[19] GonzÁlez-Jiménez, E., Xarles, X. On symmetric square values of quadratic polynomials, Acta Arithmetica 149, 145-159 (2011).

[20] GonzÁlez-Jiménez, E., Xarles, X. Five squares in arithmetic progression over quadratic fields, preprint arXiv: 0909.1663

[21] Hyndri, M., Silvermann, J.H. Diophantine Geometry, An introduction. Graduate Texts in Mathematics 201. Springer-Verlag, New York, 2000.

[22] Lang, S., TAte, J. Principal homogeneous spaces over abelian varieties, Amer. J. Math. 80, 659-684 (1958).

[23] Lazarsfeld, R. Lectures on Linear Series, With the assistance of Guillermo Fernández del Busto. IAS/Park City Math. Ser., 3, Complex algebraic geometry (Park City, UT, 1993), 161-219, Amer. Math. Soc., Providence, RI, 1997.

[24] Li, P. And Yau, S.T. A new conformal invariant and its applications to the Willmore conjecture and the first eigenvalue of compact surfaces. Invent. math. 69 (1982), 269-291.

[25] Lubotzky, A. Discrete groups, expanding graphs and invariant measures. Progress in Math. 125, Birkaüser 1994.

[26] Manin, Y. A uniform bound for p-torsion in elliptic curves. Izv. Akad. Nauk. CCCP 33, 459-465 (1969)

[27] Merel, L. Bornes pour la torsion des courbes elliptiques sur les corps de nombres. Invent. Math. 124 (1996), no. 1-3, 437-449.

[28] Mohar, B. Eigenvalues, diameter, and mean distance in graphs, Graphs Combin. 7 (1991) 53-64.

[29] Poonen, B. Gonality of modular curves in characteristic p. Math. Res. Lett. 14 (2007), no. 4, 691-701.

[30] Silvermann, J.H. The Arithmetic of Dynamical Systems, Graduate Texts in Mathematics 241, Springer-Verlag, 2007.

[31] Xarles, X. Squares in arithmetic progression over number fields, J. Number Theory 132 (2012) 379-389

[32] Zograf, P. Small eigenvalues of automorphic Laplacians in spaces of cusp forms. Zap. Nauchn. Sem. Leningrad. Otdel. Mat. Inst. Steklov (LOMI) 134 (1984), 157-168; translation in Journal of Math. Sciences 36, Number 1, 106-114

Departament de Matemàtiques, Universitat Autònoma de Barcelona, 08193 Bellaterra, Barcelona, Catalonia

E-mail address: xarles@mat.uab.cat 